\title{
Zur Auswertung von Talauenprofilen in Nordwestdeutschland
}

\author{
Von H. NiETsch, Loccum bei Wunstorf
}

$\mathrm{Z}$ us ammenf assung. Fehlerquellen bei der Auswertung horizontartig auftretender archäologischer Funde in bezug auf Lage und Zeitstellung älterer Talauenoberflächen werden besprochen und verschiedene Möglichkeiten der Auffassung von holzführenden Horizonten erläutert. Die Ergebnisse pollenanalytischer und einiger neuerer archäologischer Befunde führen zur Berichtigung älterer Anschauungen über die nacheiszeitliche Entwicklung des Wesertales.

Die nacheiszeitliche Entwicklung der größeren Täler des nordwestdeutschen Flachlandes erhält weit ïber die nächstliegenden Fragestellungen hinaus Bedeutung in der Alluvialforschung dieses Raumes durch die Beziehung auf die postglaziale Transgression im südlichen Nordseebecken. Diese Verknüpfung bringt aber auch die Gefahr weitreichender Deduktionen aus noch nicht gesicherten Grundlagen mit sich. Von den Tälern der drei Hauptflüsse unseres Gebietes ist das der W e s e r namentlich durch die Untersuchungen von E. NATERMANN in Verbindung mit den Ergebnissen im Küstengebiet von H. SCHÜтTE zum Mittelpunkt anregender Betrachtungen über die Zusammenhänge der Aufschüttungsvorgänge im Flußtal mit den Meeresspiegeländerungen geworden. Das grundlegende Verdienst beider Autoren bleibt unberührt, auch wenn die weitere Forschung in der Auffassung des seither vermehrten Beobachtungsstoffes andere Wege einschlägt.

Das Folgende soll, vom Beispiel der Weser ausgehend, unter allgemeinem Gesichtspunkt die Brauchbarkeit der Aussage einiger Beobachtungen prüfen, die gern zu Schlüssen auf die postglaziale Talentwicklung herangezogen werden. Vereinfacht gesehen, findet diese ihren Ausdruck in der jeweiligen Höhenlage der Talaue, bezogen auf die jetzige Oberfläche. Die so gestellte Frage bildet einen wichtigen Richtpunkt der einschlägigen Untersuchungen. Mit ihr verbindet sich an der Weser und anderen Flüssen die Diskussion über Alter und Bildungsbedingungen der die 'Talablagerungen nach oben abschließenden Auenlehmdecke.

Für die zeitliche Einordnung der Entwicklungsstufen steht seit längerem die Pollenanalyse zur Verfügung. Mit ihr lassen sich $\mathrm{C}^{14}$-Bestimmungen verbinden, soweit es gelingt, die in den lockeren jungen Aufschüttungen mit ihren jahreszeitlich stark wechselnden Grundwasserständen wohl in erhöhtem Maß bestehenden Fehlerquellen zu vermeiden. Zur Hauptsache stiitzten sich aber die bisherigen Vorstellungen über den Werdegang des Wesertals auf archäologisch bestimmbare Funde aus der zweiten Hälfte der Nacheiszeit, einschließlich solcher aus historischer Zeit. Mit nur ganz seltenen Ausnahmen handelt es sich hierbei um Streufunde, zumeist Tonscherben, die in Ton- und Kiesgruben in der Regel vom Bagger zutage gefördert werden, was bei tieferer Fundlage genauere Beobachtungen erschwert oder ausschließt. In mehreren Fällen wurden Einbäume geborgen.

Die Möglichkeit, solche Funde auf die ehemalige Lage der Talaue auszuwerten, wird leicht überschätzt. Treten sie vornehmlich in einer bestimmten Tiefenlage auf - nach NAtermanN (1939/40) an der Weser unterhalb von Verden etwa 7 bis $8 \mathrm{~m}$ unter der heutigen Talaue - , so kann das leicht eine flächenhafte Auflagerung und damit eine alte Taloberfläche in der vorherrschenden Fundtiefe vortäuschen, was dann auch den Versuch nahelegt, die Dauer ihres Bestehens aus den Funden abzuleiten. Ein solcher Schluß ist aber nicht ausreichend gesichert. Beschränkt sich die Fundverteilung auf die ungefähre Flußbettiefe, wie an der Weser mindestens von der frühen Bronzezeit ab, so wird es sogar sehr wahrscheinlich, daß die Talaue während der ganzen archäologisch belegten Zeit schon annähernd dieselbe Höhe wie heute gehabt hat. Die Gegenstände können in irgendwelchen Altwassern, aber auch an der Sohle des lebenden, mäandrierenden Flusses ein- 
gebettet worden sein. Letztere Annahme kommt der überwiegend gleichen Tiefenlage von Funden ganz verschiedener Zeitstellung am besten entgegen. Die nicht selten in derselben Tiefe beobachtete Einstreuung von Blättern, Holzstückchen und kleinen Knochenteilen (Natermann a.a.O.) fügt sich dem aufs beste ein; auch dic vereinzelt gute Erhaltung von Tongefäßen ist unter Wasser leichter denkbar als an der Oberfläche, wo sie Schädigungen durch Frost, Pflanzenwurzeln und andere Einwirkungen viel mehr ausgesetzt gewesen wären.

Auf jeden Fall dürfen die bisherigen Auffassungen von einer bis in das erste nachchristliche Jahrtausend beträchtlich tieferen Lage der Wesertalaue oberhalb von Bremen und ihrer schnellen Aufschüttung unter dem Einfluß des steigenden Meeresspiegels in den folgenden Jahrhunderten durch die Ergebnisse pollenanalytischer Untersuchungen als endgültig widerlegt gelten (s. H. Nietsci 1955 a, b, 1959, z. T. in ergänzender Auswertung älterer Pollenzählungen und Profilangaben aus der Wümmeniederung bei Bremen von F. Overbeck in Overbeck \& Schmitz 1931, S. 49 ff.). Damit decken sich die Aussagen der wenigen archäologischen Befunde, dic klare Schlïsse auf die Lage der entsprechenden Oberfläche zulassen; hier sind vor allem, außer Grabungsergebnissen aus dem Untergrund der Altstadt Bremens von K. H. BRANDT, Urnenbeisetzungen wenige Dezimeter unter der heutigen Oberfläche der Talaue bei Wellie zu nennen ${ }^{1}$ ).

Das so berichtigte Bild wird auch durch Feststellungen an der Ems bestätigt, deren Talaue am Unterlauf, nördlich von Leer bei Jemgum, seit dem Ende der Bronzezeit nur um etwa ein Meter angewachsen ist (W. HA.tRNAGEL 1957), während weiter südlich, zwischen Dörpen und Meppen - was an der Weser etwa dem Talabschnitt unterhalb der Allermündung entspräche - eine zeitlich vergleichbare allgemeine Aufschüttung offenbar überhaupt fehlt (H. MüLlER 1956).

Diese gut übereinstimmenden neueren Feststellungen an Weser und Ems machen eine Überprüfung auch der an der E 1 b e angenommenen jungalluvialen Aufschüttungen wünschenswert. Nach Ausscheidung aller nicht einwandfrei auf eine Erhöhung der Talaue abgesehen von dem Absatz von Hochwasserlehmen - beziehbaren Unterlagen dürfte sich auch dort bestätigen, daß die Begleiterscheinungen des nacheiszeitlichen, mehr oder minder eustatischen Meeresanstiegs im wesentlichen auf den unter Gezeiteneinfluß stehenden unteren Flußlauf beschränkt geblieben sind (vgl. NIETSCH 1952).

Die gleichen Einwände, die der Auswertung archäologischer Belege aus den Flußablagerungen oft entgegenstehen, gelten auch für die Beurteilung der nicht seltenen Ansammlungen von Holztrümmern und ganzen Baumstämmen in den Talablagerungen, der sogenannten "Rannenhorizonte" nach einer süddeutschen Bezeichnung (Н. JАков 1956). Auch hierbei muß mit mehreren Möglichkeiten gerechnet werden. Offenbar handelt es sich in den meisten Fällen um Zusammenschwemmungen, die in Nebenarmen des Flusses steckengeblieben sind, wonach das Holz schließlich auf den Grund sank. Solche Erscheinungen können ein riesiges Ausmaß annehmen, wie Schilderungen des „Red-River-Floßes“ aus den Entdeckerzeiten Nordamerikas erkennen lassen (J. BaKeless). Daneben kam wohl mehr vereinzelt die Einbettung untergesunkener Stämme an der Sohle des lebenden Flusses vor. Es ist aber nicht zu bezweifeln, daß in noch waldreichen Zeiten des ungeregelten Abflusses die Hochwässer regelmäßig treibende Stämme auch auf der Talaue

1) Die schon (NIETsCh 1955a, S. 32) mitgeteilten Fundumstände seien noch durch eine genauere Angabe vervollständigt, die ich nachträglich von einem Fundzeugen erhielt: danach wurde man bei dem üblichen Abräumen der humosen Bodenschicht über dem Ziegelton auf eine rötliche, auf Feuereinwirkung deutende Verfärbung an dessen Oberfläche aufmerksam und stieß bei weiterer Untersuchung schon etwa $1 \mathrm{dm}$ tiefer auf die Urnen. Unter der so gut wie sicheren Voraussetzung, daß beides zeitlich zusammengehört, besteht demnach hein $Z$ weifel, daß die Tongefäße in den Lehm eingegraben, nicht ihm aufgesetzt worden sind. Seit den Bestattungen um den Beginn unserer Zeitrechnung hat sich also an der noch in der Gegenwart bei größeren Hochwassern überfluteten Fundstelle die Oberfläche der Talaue nur um etwa $40 \mathrm{~cm}$ erhöht. 
absetzten oder die Spuren katastrophenartiger Verwüstungen von Auenwäldern an Ort und Stelle zurückließen. In diesen Fällen ist es aber wenig wahrscheinlich, daß die der Taloberfläche aufgelagerten Hölzer erhalten blieben. Selbst bei schnell fortgeschrittener Aufschüttung, bei der an mehr oder minder grobe Sedimente zu denken wäre, würde dieses Holz normaler Weise den größten Teil des Jahres über dem Grundwasser entzogen gewesen sein. Die oft ausgezeichnete Erhaltung der noch gern genutzten Stämme spricht aber für ständige Wasserbedeckung.

Selbst aufrechte Wurzelstubben sind nicht ohne weiteres Belege einer alten Oberfläche, es sei denn, daß die Verwurzelung im Boden festzustellen ist oder nach sonstigen

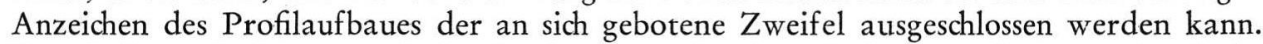
Der tiefliegende Schwerpunkt begünstigt ein senkrechtes Aufsetzen schwimmender Stubben, wovon man sich gelegentlich am Ufer von Waldgewässern überzeugen kann. Recht aufschlußreich ist in dieser Beziehung die eindrucksvolle Beschreibung, die A. E. v. NorDENSKiöld (1921), der Bezwinger der Nordostdurchfahrt, von der Jenisseimündung gab. Nach Erwähnung der großen Treibholzmengen, die der Strom dem Meere zuführt, fährt der Bericht fort: „Die Hauptmasse dieses Holzes bleibt nicht am Ufer liegen, sondern schwimmt in die See hinaus, um dort in den Meeresströmungen zu treiben, bis es sich mit Wasser vollgesogen hat und sinkt. Ein Teil dieser sinkenden Riesenstämme hatte sich senkrecht in den Sand des Hafens eingepflanzt, und eine Bucht in der Nähe des Dicksonhafens war durch ein Palisadenwerk von Treibholzstämmen vollkommen gesperrt.“

Manche andere Wirkungen der elementaren Naturvorgänge, vor allem der Hochwasser, lassen sich auch unter den veränderten Verhältnissen des Kulturlandes wenigstens mittelbar noch aus dem heutigen Geschehen ableiten. Eine eigenartige Erscheinung sind beispielsweise an der Elbe wassergefüllte Hohlformen, von denen die kleineren riesigen Bombentrichtern gleichen, die größeren, unregelmäßiger geformten, schon richtige kleine Seen darstellen. Diese Ausräumungen, die sogenannten „Bracks“, haben genetisch mit den Altwassern stillgelegter und verlandender Flußläufe nichts zu tun, sie sind die Folge von Deichbrüchen. Die Bracks und sie fächerartig umgebende flache Sandauflagerungen über der ursprünglichen Oberfläche zeugen von der Gewalt der im Katastrophenfall den Deich durchbrechenden aufgestauten Wassermengen. Auch im natürlichen Hochwassergeschehen, besonders wohl auf bewaldeten Talauen, stellten sich dem Abfluß Hindernisse entgegen, zusammengetriebene Eisschollen, Holz und anderes, die zu ähnlichen Durchbrüchen mit örtlichen Auskolkungen Anlaß gaben. Von L. HEMPEL (1956) aus dem Leinetal bei Göttingen beschriebene Profile lassen sich gut als Ausfüllung einer solchen mit schnell wechselnden Strömungsrichtungen der Wassermassen verbundenen Erosion auffassen. Deshalb ist Vorsicht geboten bei der Verallgemeinerung der Schliisse auf bestimmte Phasen der Auenlehmbildung.

\section{Schriften-Verzeichnis}

Bakeless, J.: The Eyes of Discovery. The pageant of North America as seen by the first explorers (Ubersetzung: "Schimmernde Berge und weite Prärien" von H. G. Sommerwerck, G. STAlling, Oldenburg i. Oldbg.).

HraRnageL, W.: Die spätbronze-früheisenzeitliche Gehöftsiedlung Jemgum bei Leer auf dem linken Ufer der Ems. - Die Kunde, Mitt. nieders. Landesver. Urgesch. N.F. 8, S. 2-44, Hannover 1957.

Hempel, Ludwig: Über Alter und Herkunftsgebiet von Auelehmen im Leinetal. - E. u. G. 7, S. $35-42,1956$.

HövermanN, J.: Studien über die Genesis der Formen im Talgrund südhannoverscher Flüsse. Nachr. Akad. Wissensch. Göttingen, math.-phys. Kl. IIb, S. 1-14, 1953.

ЈАКов, H.: Zur Datierung des „Rannenhorizontes“ und der sog. „Pfahlbauten“ im Main-RegnitzGebiet um Bamberg. - Ber. natf. Ges. Bamberg 35, S. 1-20, 1956.

Mensching, H.: Die Entstehung der Auelehmdecken in Nordwestdeutschland. - Proceed. III. Intern. Congr. Sedimentology, Groningen-Wageningen, 1951. 
MüLLER, H.: Ein Beitrag zur holozänen Emstalentwicklung zwischen Meppen und Dörpen auf Grund von pollenanalytischen Untersuchungen. - Geol. Jb. 71, S. 491-504, Hann. 1956.

Natermann, E.: Zur Geologie der Wesermarsch oberhalb Achim. - Abh. natw. Ver. Bremen 31, S. $154-167,1939 / 40$.

NiEtsch, H.: Zur spät- und nacheiszeitlichen Entwicklung einiger Flußtäler im nordwestlichen Deutschland. - Z. deutsch. geol. Ges. 104, S. 29-40, Hannover 1952. - - Hochwasser, Auenlehm und vorgeschichtliche Siedlung. - Erdkunde 9, S. 20-39, Bonn 1955 (a). - Untersuchungen über die jüngere Talgeschichte der Weser bei Schlüsselburg und das Alter des Niederterrassenlehms bei Stolzenau. - Jb. geogr. Ges. Hannover für 1954 und 1955, S. 19-28, Hannover 1955. (b) - - Pollenanalytischer Beitrag zur Geschichte der Wesermarsch bei Bremen. - Die Kunde N. F. 9 (1958), S. 72-83, Hannover 1959.

Nordenskiöld, A. E. Frh. von: Die Umsegelung Asiens und Europas auf der Vega. - Reisen und Abenteuer 9, Leipzig (F. A. Brockhaus) 1921.

Overbeck, F. \& Schmitz, H.: Zur Geschichte der Moore, Marschen und Wälder Nordwestdeutschlands. I. - Mitt. Prov. St. Naturdenkmalpfl. Hannover 3, S. 1-179, 1931.

Sснӥтte, H.: Sinkendes Land an der Nordsee. - Schr. dtsch. Natkdever. N. F. 9, 1939.

Manuskr. eingeg. 27. 2. 1959.

Anschrift des Verf.: Dr. H. Nietsch, (20a) Loccum über Wunstorf, Nr. 243. 